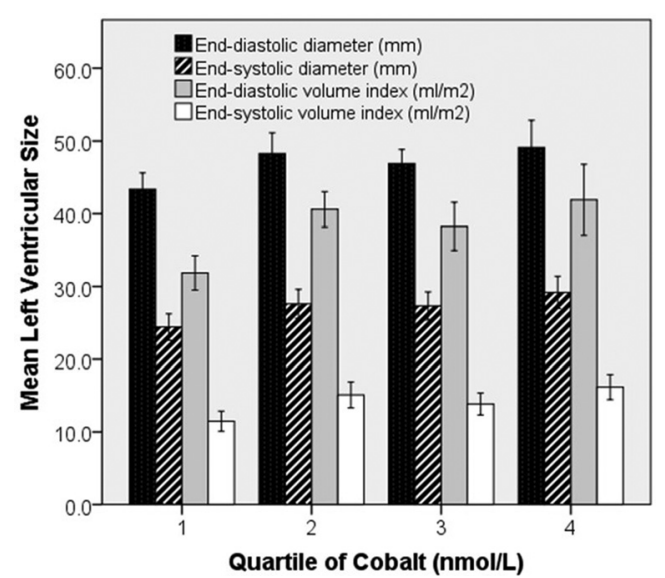

Abstract 15 Figure 1 Mean left ventricular chamber size by quartile of serum cobalt levels. Bars represent standard error.

squared tests were used to evaluate the differences between groups.

Results Baseline characteristics between each quartile were similar (table 1). Mean duration of implant increased with quartile, but serum cobalt was not correlated with duration of prosthesis $(r=0.135, p=0.162)$. Patients in the highest quartile of serum cobalt levels had larger left ventricular end-diastolic and end-systolic dimensions (50.8 vs. $44.7 \mathrm{~mm}, \mathrm{p}=0.018$; 31.6 vs. $25.8 \mathrm{~mm}, \mathrm{p}=0.014$ respectively) and indexed end-diastolic and end-systolic volumes by Simpsons method (42.0 vs. $32.8 \mathrm{ml} / \mathrm{m}^{2}, \mathrm{p}<0.001$; 16.4 vs. $11.7 \mathrm{ml} / \mathrm{m}^{2}, \mathrm{p}<0.001$ respectively) compared with those in the lowest quartile (Figure). They also had larger indexed left atrial volumes (Ln volume 3.2 vs. $2.9, \mathrm{p}=0.006)$. However, there was no difference in wall thickness, ejection fraction, left ventricular outflow tract velocity time integral, mitral valve $\mathrm{E}$ and $\mathrm{A}$ waves, medial and lateral tissue Doppler magnitude, peak longitudinal strain or strain rate, or time to peak strain.

Conclusions Subjects with the highest serum cobalt levels have larger left ventricular and left atrial chamber sizes than those with the lowest serum levels. However, no difference in even sensitive markers of systolic or diastolic function were found.
Left ventricular measurements are part of a range and therefore it is not possible to specify a cut-off for abnormality in these people. Whilst our findings do not suggest a clear relationship between serum ion levels and basic echocardiographic parameters of left ventricular systolic and diastolic dysfunction, we cannot exclude an idiosyncratic response as described in isolated case reports. We therefore advise that patients with MOM hip replacements undergo serial cardiology follow up.

\section{CHRONOTROPIC AND DROMOTROPIC RESPONSES TO EXERCISE IN HEART FAILURE WITH PRESERVED EJECTION FRACTION (HFPEF) PATIENTS AND CONTROLS}

${ }^{1}$ Freya Lodge*, ${ }^{2}$ Tamas Erdei, ${ }^{3}$ Heather Edwards, 'Zaheer Yousef, ${ }^{1}$ Alan Fraser. ${ }^{1}$ Cardiff and Vale University Health Board; ${ }^{2}$ Bristol Heart Institute; ${ }^{3}$ Aneurin Bevan Health Board

\subsection{6/heartjnl-2017-311726.16}

Introduction The hallmark of Heart Failure with Preserved Ejection Fraction (HFpEF) is exercise intolerance. The mechanisms for this are numerous, but chronotropic incompetence, defined as a failure to reach at least $70 \%$ of the age-predicted maximum heart rate (HR) on maximal exercise, has been reported to contribute. Impaired Heart Rate Reserve, a measure of HR achieved on maximal exercise compared with agepredicted maximum heart rate, is correlated with negative cardiovascular outcomes.

In normal subjects, the PR interval shortens during exercise as parasympathetic tone reduces. This is known as dromotropy and is reduced in subjects with coronary artery disease. We formed the hypothesis that HFpEF patients may also have impaired PR shortening and that this may contribute to exercise intolerance.

Methods and results HFpEF patients and controls (healthy $(\mathrm{H})$, hypertensive (HT) and breathless controls (BC)) from the MEDIA (Metabolic Road to Diastolic Heart Failure, EU FP7) trial at our centre underwent maximal semi-supine bicycle stress tests whilst on standard treatment. Electrocardiograms were examined by a single, blinded investigator for PR-interval and heart rate (HR) at: rest; submaximal exercise (HR $100 \mathrm{~min}-1)$; peak exercise; and 2 and $5 \mathrm{~min}$ after exercise.
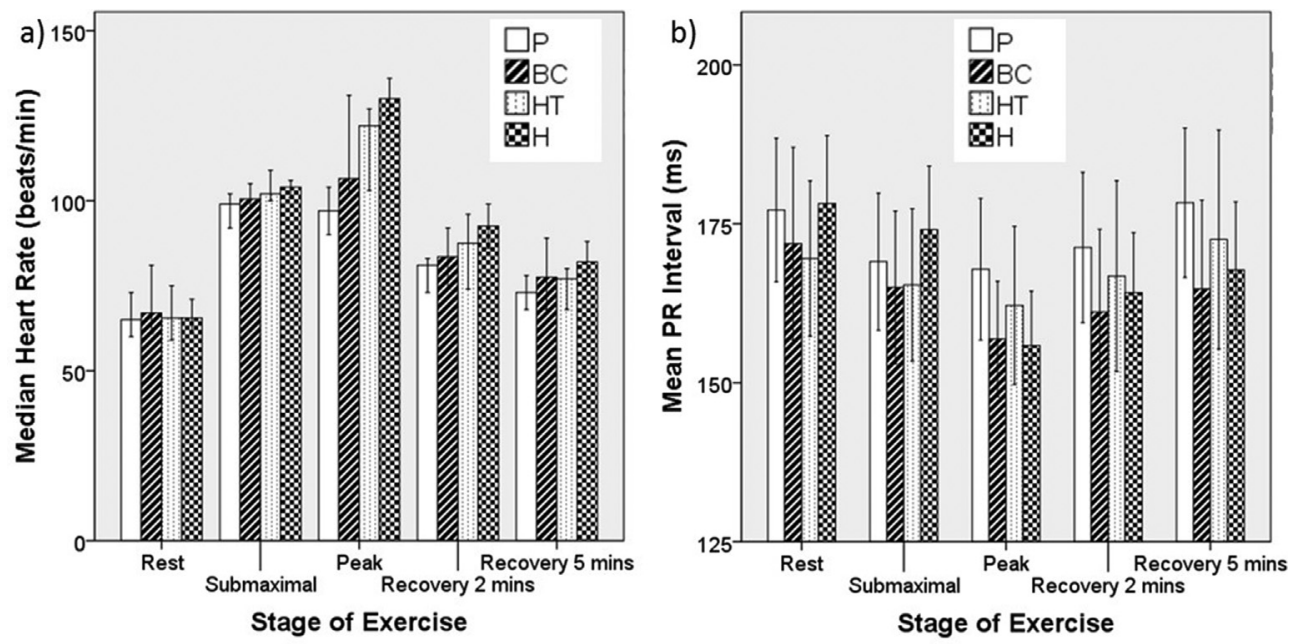

Abstract 16 Figure 1 a) Median heart rate and b) Mean PR interval by stage of exercise for each patient group. Bars represent $95 \%$ Confidence Interval. $\mathrm{P}=$ Patients, $\mathrm{BC}=$ Breathless Controls, $\mathrm{HT}=$ Hypertensive Subjects, $\mathrm{H}=$ Healthy Controls. 

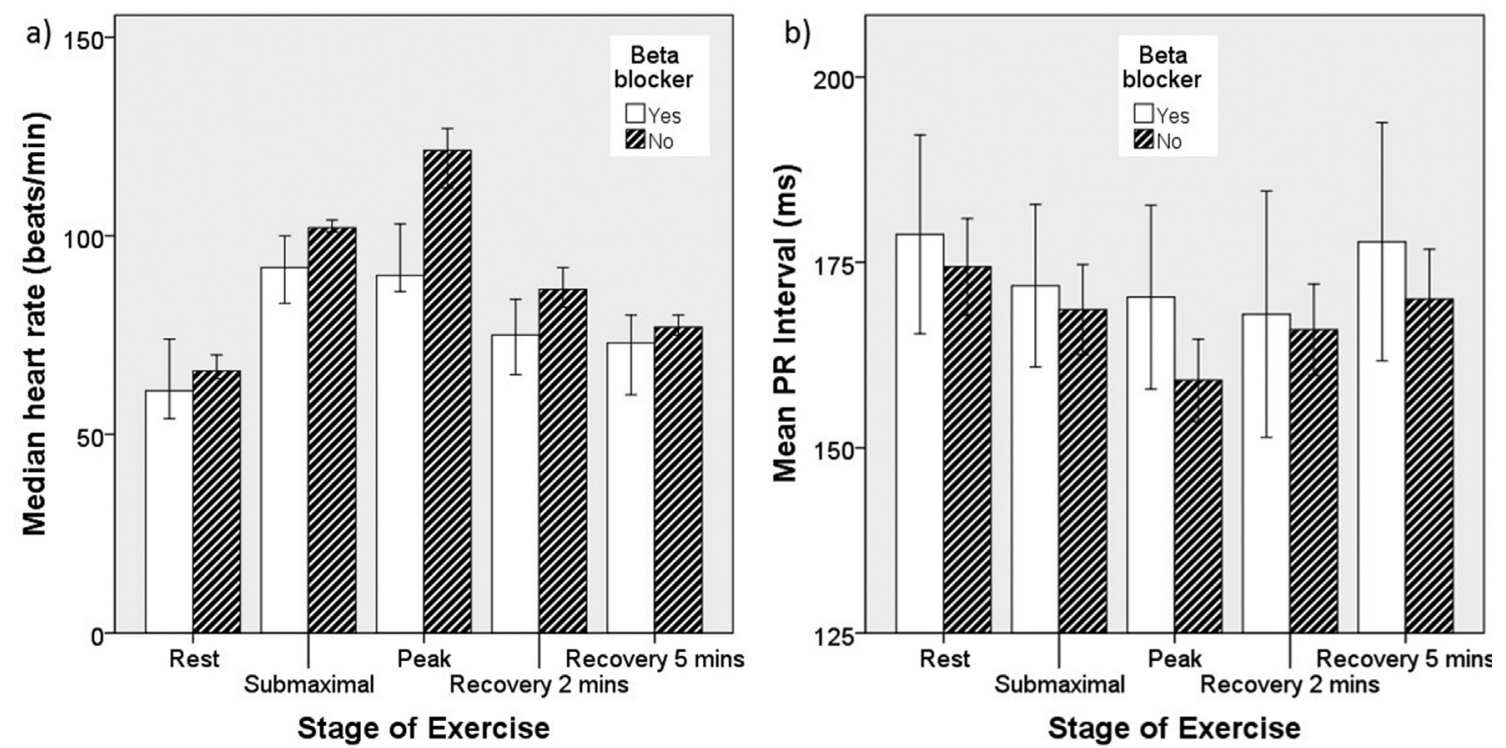

Abstract 16 Figure 2 Effect of beat blockers on a) heart rate and b) PR interval by stage of exercise. Bars represent $95 \%$ confidence limits.

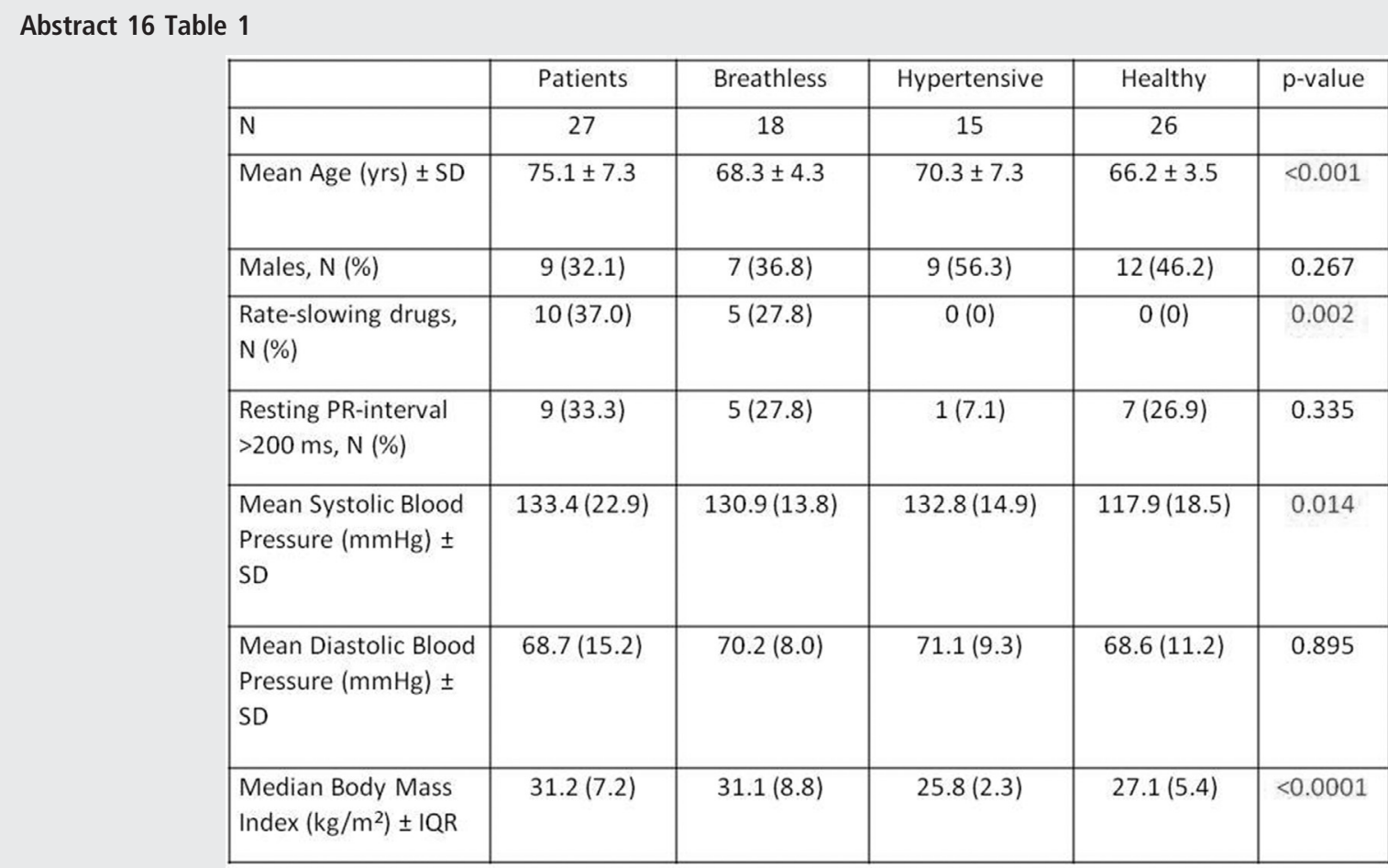

110 subjects were recruited, of whom 24 were excluded (unable to exercise/atrial arrhythmia). Data on 86 subjects were analysed. Baseline characteristics are given in the Table.

Resting HR was similar between groups, but maximal HR was lower in patients than all controls $(\mathrm{p}<0.05)$, as was HR $(\mathrm{p}<0.0001)$ (figure 1). HR recovery was impaired in patients versus healthy controls at $2 \mathrm{~min}(\mathrm{H}=-34.8, \mathrm{p}<0.001)$ and $5 \min (\mathrm{H}=-17.9, \mathrm{p}=0.04)$ of recovery. PR interval decreased across all groups from rest to peak (mean $-7.2 \%, \mathrm{p}=0.025$ ).
PR interval was similar at rest; it shortened less in patients $(5.2 \%)$ versus healthy controls $(11.4 \%)$, but not when corrected for difference in peak $\mathrm{HR}$. PR/HR did not vary by group at any stage. Beta blockers did not affect PR interval, but reduced $\mathrm{HR}$ and $\mathrm{HR}$ at all stages $(35 \%$ reduction from rest to peak) (figure 2). The difference in maximal HR between patients and controls persisted after excluding beta blocked subjects. 
Patients were $13 \%$ older than controls $(p<0.01)$. Subjects from all groups of less than 70 years had significantly increased PR shortening (mean difference $-9.1 \mathrm{~ms}, \mathrm{p}=0.02$ ) and change in HR (mean difference $15.7 \mathrm{~ms}, \mathrm{p}<0.001$ ) between rest and peak than those over 70 years. Patients had significantly higher Body Mass Indices (BMI) than hypertensive or healthy subjects $(\mathrm{H}=-3.6, \mathrm{p}=0.02, \mathrm{H}=-3.5, \mathrm{p}=0.003$ respectively), as did breathless controls $(\mathrm{H}=3.1, \mathrm{p}=0.012$, $\mathrm{H}=2.8, \mathrm{p}=0.027$ ).

Conclusions Patients with HFpEF have chronotropic incompetence and impaired HR recovery, but there was no evidence of dromotropic incompetence in this study.

\section{OUTCOMES OF RECIPIENTS WITH NON-ISCHAEMIC HEART DISEASE AFTER HEART TRANSPLANTATION}

Maria Simonenko*, Yulia Sazonova, Petr Fedotov, Vadim Rubinchik, Tatjana Pervunina, Aelita Berezina, Kirill Malikov, Andrey Bautin, Vladimir Krasnov, Vladimir Privorotskii, Dmitry Zverev, Lubov Mitrofanova, Maria Sitnikova, German Nikolaev, Michail Gordeev, Michail Karpenko. Federal Almazov North-West Medical Research Centre

\subsection{6/heartjnl-2017-311726.17}

Objective The aim of study was to estimate outcomes of recipients with non-ischaemic chronic heart failure (CHF) after heart transplantation (HT).

Methods From 2010 to 2016 we heart transplanted 80 recipients: $52.5 \%(\mathrm{n}=42)$ - with non-ischaemic CHF (mean age $38.3 \pm 2,2$ years, $\mathrm{n}=4$ children (mean age $-13.5 \pm 2.6$ years)), $47.5 \%(\mathrm{n}=38)$ - with ischaemic heart disease (IHD). Causes of CHF (mean LVEF - 20.9 $1.8 \%$ ) in non-ischaemic group: dilated cardiomyopathy $(61.9 \%, \mathrm{n}=26)$, non-compacted myocardium $(21.4 \%, n=9)$, rheumatic heart disease $(4.8 \%, n=2)$, arrhythmogenic right ventricular dysplasia $(7.1 \%, \mathrm{n}=3)$ and others ( $n=1$ cardiac amyloidosis, $n=1$ cardiac sarcoidosis). Furthermore, 19\% $(\mathrm{n}=8)$ of them were supported by biventricular assist device Berlin Heart EXCOR (duration on support $234 \pm 80$ days) prior HT. According to right heart catheterization results: pulmonary arterial systolic pressure (PASP) 39.9 $\pm 1.9 \mathrm{mmHg}$, pulmonary vascular resistance (PVR) -2.9 \pm 0.2 W.U. Pulmonary hypertension was diagnosed in $66.7 \%$ $(n=28)$ recipients. There was no difference in how much time patients spent in HT waiting list $(158.9 \pm 23.8$ days vs. 152.4 \pm 29.4 days, $p>0.05$ ). All recipients treated by triple-drug therapy (steroids, calcineurin inhibitors, mycophenolic acid), induction: basiliximab - 62\% ( $\mathrm{n}=26)$, thymoglobulin - $38 \%$ $(n=16)$. Outcomes of HT were estimated by survival, TTE results, frequency of rejection, frequency of cardiac allograft vasculopathy (CAV) and cardiopulmonary exercise test results.

Results After 6 year follow-up survival was comparable with IHD recipients (30.4 $\pm 3,1$ months vs. $27.3 \pm 4.2$ months, $\mathrm{p}>0.05$ ), but mortality during 1 st $\mathrm{yr}$ after HT was higher in IHD group $(7.1 \%$ vs. $13.1 \%, \mathrm{p}<0.001)$. After HT non-ischaemic patients spent less time in ICU $(9.3 \pm 0.9$ days vs. 11.5 \pm 1.6 days, $\mathrm{p}>0.05)$, the same as required inotrope support $(6.1 \pm 0.6$ days vs. $8.5 \pm 1.4$ days, $\mathrm{p}<0.05)$. Only 9.5\% $(\mathrm{n}=4)$ of recipients had severe right heart failure and required extracorporeal membrane oxygenation (ECMO) support, 7\% $(n=3)$ - underwent tricuspid valve repair due to severe tricuspid regurgitation. Levosimendan treatment was successfully used in $33.3 \%(n=14)$ patients. In 1 case thoracoscopic pericardial fenestration performed. In 6 months after HT TTE results got to normal values: LVEF - 60.6 $\pm 0.9 \%$, PASP $31.1 \pm 1.3 \mathrm{~mm} \mathrm{Hg}$; results were stable even in 1 year. During 1st yr rejection was found: $\mathrm{R} 2 / 3 \mathrm{~A}-9.5 \%(\mathrm{n}=32)$, AMR1 $2.1 \%(n=7)$. Also in 1 year after HT recipients from nonischaemic group significantly improved their physical capacity $\left(\mathrm{V0}_{2 \text { peak }}-11.7 \pm 0.4 \mathrm{ml} / \mathrm{min} / \mathrm{kg} \quad\right.$ vs. $\quad 17.8 \pm 0.6 \mathrm{ml} / \mathrm{min} / \mathrm{kg}$, $\mathrm{p}<0.001)$. CAV was diagnosed in more than 1 year of followup. Furthermore, frequency of CAV was more often in IHD group $(9.5 \%(n=4)$ vs. $13.2 \%(n=5), p<0.05)$.

Conclusion After HT survival and complications are comparable in recipients with IHD and non-ischaemic group.

\section{UPGRADE TO DEFIBRILLATOR IN PATIENTS RECEIVING CARDIAC RESYNCHRONISATION THERAPY PACEMAKERS}

${ }^{1}$ Shveta Monga*, 'Dominic Haigh, 'David Royan, ${ }^{1}$ Paul Foley, ${ }^{1}$ Badrinath Chandrasekaran. ${ }^{1}$ Great Western hospital NHS trust

\subsection{6/heartjnl-2017-311726.18}

Introduction In patients with advanced cardiac failure, the choice between CRT with (CRT-D) or without a defibrillator (CRT-P), remains a contentious issue. There is insufficient evidence from randomised controlled trials and guidelines do not make any firm recommendations. This leaves room for physician discretion and has led to wide variation in worldwide practice.

Study aim and basic methods The focus of our study was to evaluate the characteristics of CRT-P patients in the real world setting and analyse how many patients with CRT-P implant required an upgrade to CRT-D in the follow-up period.

This was a retrospective single centre observational study looking at 122 patients implanted with CRT from 2011 to 2015. Data was collected from patient electronic records and follow-up visits. The median follow-up duration was 2.2 (1.23.3) years. Data was analysed using Microsoft excel and SPSS. Results 1. The patients implanted with CRT-P were elderly, had more advanced heart failure with multiple co-morbidities and mostly females.

2. Only 3 out of 122 patients (2.5\%) were upgraded from CRT-P to CRT-D. All these patients were males, above 75 years of age with a primary prevention indication for CRT-D and had upgrade following episodes of symptomatic ventricular tachycardia (Figure 1).

\begin{tabular}{|l|l|l|}
\hline & Upgrade & CRT-P \\
\hline Number of patients & 3 & 122 \\
\hline Mean Age in yrs & 77.3 & 79.2 \\
\hline Female & None & $24 \%$ \\
\hline Ischaemic heart disease & $33 \%$ & $48 \%$ \\
\hline Dilated cardiomyopathy & $67 \%$ & $51 \%$ \\
\hline
\end{tabular}

Abstract 18 Figure 1 Clinical profile of upgraded patients compared to rest of the CRT-P population

3. The main cause of mortality in patients implanted with CRT-P was non-cardiac vs. cardiac $(5.7 \%$ vs. $15.6 \%$, p value $<0.001)$. Majority of deaths in the cardiac group were due to pump failure. Only $10 \%$ of patients in this group had documented non sustained ventricular arrhythmias.

Conclusion In our study population, the number of CRT-P patients who needed upgrade with additional defibrillator was 